\title{
Management of Eclampsia
}

\section{DEFINITION}

Eclampsia is defined as the development of convulsions and/or unexplained coma during pregnancy or postpartum in patients with features of preeclampsia.

\section{DIAGNOSIS}

- Hypertension is considered the hallmark for the diagnosis of eclampsia. However, in $16 \%$ of the cases hypertension may be absent.

- Eclampsia is usually associated with proteinuria, but this may be absent in $14 \%$ of cases.

- Clinical features of imminent eclampsia include:

- Severe frontal headache,

- Visual symptoms (halos, scotomas etc.)

- Epigastric or right hypochondria I pain,

- Liver tenderness,

- Clonus (3 beats or more)

\section{TIME OF ONSET OF ECLAMPSIA}

The onset of eclamptic convulsions can be antepartum, intrapartum, or postpartum.

\section{Antepartum eclampsia}

Almost all cases (91\%) develop eclampsia at or beyond 28 weeks.

\section{Postpartum eclampsia}

Although most cases of postpartum eclampsia occur within the first 48 hours, some cases develop beyond 48 hours, up to 4 weeks postpartum (late postpartum eclampsia). In these cases, an extensive neurological evaluation is needed to rule out the presence of other cerebral pathology.

\section{COMORBIDITIES}

- Eclampsia is often complicated by comorbidities (Box 1).

\begin{tabular}{|ll|}
\hline \multicolumn{2}{|c|}{ Box 1 } \\
\hline - & \multicolumn{2}{c|}{ Abruptio placentae } \\
- & Disseminated intravascular coagulopathy \\
- & Pulmonary oedema \\
- Acute renal failure & Aspiration pneumonia \\
- HELLP syndrome (Haemolysis, elevated liver \\
enzymes, low platelets)
\end{tabular}

- These are more common among women who develop eclampsia at earlier periods of gestation.

\section{PREVENTION}

Administration of magnesium sulphate to women with features of impending/imminent eclampsia (presence of clonus, severe headache, visual disturbances, dizziness) is the only known preventive measure.

\section{MANAGEMENT}

\subsection{General considerations}

6.1.1 The priorities in management are to support respiratory and cardiovascular function, prevent injury and further seizures and to control hypertension.

6.1.2 Magnesium sulphate is the anticonvulsant of choice. It must be administered as soon as possible. See section 6.2.2 of the severe preeclampsia guideline for details.

6.1.3 The bolus dose of magnesium sulphate must be given even to women with unknown renal function or oliguria/anuria since this dose is unlikely to elevate magnesium levels to toxic ranges.

6.1.4 Eclampsia dictates delivery (or induction) once the maternal condition is stabilized, irrespective of the foetal condition or maturity. A decision regarding the mode and time of delivery will require to be made early.

6.1.5 There is no place for prolongation of the pregnancy in these women, unless under rare, exceptional circumstances.

6.1.6 For details on administration of medications and intravenous fluids and care of women receiving magnesium sulphate and intravenous antihypertensives, refer the guideline on severe preeclampsia.

\subsection{During the seizureo}

Turn the patient to a side and support her in that position.

- Suck out secretions from the mouth.

- Administer oxygen via a face mask.

- Most eclamptic seizures resolve spontaneously.

- It is imprudent to diagnose fetal hypoxia based on fetal bradycardia during a seizure. This usually recovers spontaneously following the seizure.

- Fetal bradycardia persisting beyond 10 minutes 
following the seizure should raise suspicion of abruptio placentae.

\subsection{As soon as possible following a seizure}

- Attempt to establish intravenous access.

- Obtain blood for full blood count, liver transaminases, blood urea, electrolytes and blood for cross-match.

- Start magnesium sulphate (intravenous bolus and infusion or intramuscular - details in guideline on severe preeclampsia section 6.2.2.).

- Treat blood pressure as appropriate.

- Insert an indwelling catheter.

- Monitor respiratory rate, urine output, reflexes, spa,. (Please refer the guideline on severe preeclampsia for further details).

- Check for comorbidities (Box 1).

- Inform the Consultant and establish a plan of management.

\subsection{Management af seizures in wamen receiving magnesium sulphate}

6.4.1 Women developing a seizure while on magnesium sulphate

- $10 \%$ of women receiving magnesium sulphate will develop a second seizure.

- Administer magnesium sulphate 2 grams diluted to $10 \mathrm{ml}$ with $0.9 \%$ sodium chloride solution over 5 minutes.

- Increase the magnesium sulphate infusion to 2 grams per hour while monitoring as above.

6.4.2 Women developing more than one seizure while on magnesium sulphate

\section{DELIVERY}

- Call a Neurology team for advice. If one is not available, obtain advice from a medical team.

- Consultant must be informed.

- Inform the anaesthetic team if still not in an intensive care setting.

- Second line anticonvulsants must be considered after discussing with anaesthetist.

- If the woman develops further seizures, consider moving to intensive care for neuromuscular paralysis and ventilation.

- These women will require a full neurological evaluation, including imaging.

- Eclampsia is not an indication for caesarean section.

- Consider caesarean section in women who are not in labour with a Bishop score below 7.

- Women who are in labour may be allowed to continue to delivery, in the absence of obstetric complications.

- Labour may be induced where necessary using either prostaglandins or amniotomy and oxytocin infusion.

- Epidural or spinal anaesthesia may be administered in women with platelet counts above $80,000 / \mathrm{cu} \mathrm{mm}$.

- General anaesthesia is best avoided where possible since it increases the risk of aspiration and failed intubation due to airway oedema. It is also associated with marked increases in systemic and cerebral pressures during intubation and extubation. Women with airway or laryngeal oedema may require 'awake intubation' under fibre optic observation with facilities available for immediate tracheostomy. The level of increase in systemic or cerebral pressures may be reduced by pretreatment with labetalol or nitroglycerine injections.

\section{TRANSFER OF A WOMAN WHO HAS HAD A SEIZURE TO ANOTHER INSTITUTION}

- In case it is required to transfer a woman who has had an eclamptic seizure, this must be done only after administering a bolus of magnesium sulphate. (See section. 6.2.2 of the severe preeclampsia guideline for details). The patient should ideally be accompanied by a doctor and emergency drugs/equipment (e.g. Ambu bag) must be available.

\section{POSTPARTUM MANAGEMENT}

- Continue administration of magnesium sulphate and monitoring as described in the guideline on severe preeclampsia.

- Women with abnormal renal function, preexisting hypertension and abruption placentae (due to use of larger than normal volumes of fluids) are at particularly high risk of pulmonary oedema. They will require appropriate monitoring.

- Antihypertensive therapy may be changed to the oral route and continued.

\section{COUNSELLING}

\subsection{Women should be advised that in a subsequent pregnancy:}

- The rate of preeclampsia is approximately $25 \%$.

- Rate of eclampsia is $2 \%$.

- These rates are substantially higher in women who develop eclampsia in the second trimester.

- $\quad$ Taking high-dose calcium from early pregnancy (600 $\mathrm{mg}$ daily) and aspirin (75 $\mathrm{mg}$ daily) may reduce this risk. 


\subsection{Regarding long term risk of hypertension}

- There is no increase of risk in women who were normotensive before the pregnancy.

- Multigravidae who develop eclampsia may be at high risk.

\section{ACKNOWLEDGEMENT}

The following article was used as a resource in developing this guideline: Baha M Sibai, Diagnosis, Prevention and Management of Eclampsia. Obstetrics \& Gynecology, 2005; 105 (2): 402 - 410 